ORIGINAL ARTICLE

\title{
Initial effects of anti-pronation tape on the medial longitudinal arch during walking and running
}

\author{
B Vicenzino, M Franettovich, T McPoil, T Russell, G Skardoon
}

Br J Sports Med 2005;39:939-943. doi: 10.1136/bjsm.2005.019158

See end of article for authors' affiliations

....................

Correspondence to: Associate

Professor Vicenzino, Department of Physiotherapy, University of Queensland, St Lucia, Brisbane, Queensland 4072, Australia;

b.vicenzino@uq.edu.au

Accepted 9 May 2005
Objectives: To investigate the effect of an augmented LowDye taping technique on the medial longitudinal arch of the foot during dynamic tasks such as walking and jogging, and to elucidate the relation between tape induced changes in static and dynamic foot posture.

Methods: Seventeen subjects (mean (SD) age 27 (5.8) years) who were asymptomatic and exhibited a navicular drop greater than $10 \mathrm{~mm}$ were studied. Medial longitudinal arch height standardised to foot length during standing and at mid-stance of walking and jogging was measured from digital video images taken before and after the application of an anti-pronation taping technique. A no tape control condition was also included.

Results: Compared with the no tape control condition, tape produced a significant mean (SD) increase in the medial longitudinal arch height index of 0.031 (0.015), $0.026(0.014)$, and $0.016(0.017)$ during standing, walking, and jogging respectively $(p<0.05)$. The relative increase in medial longitudinal arch height represents an anti-pronation effect. The tape induced changes in the medial longitudinal arch height measured during standing correlated strongly with those measured during walking and jogging (Pearson's $r=0.7$ and 0.76 respectively).

Conclusions: The augmented LowDye tape was effective in controlling pronation during both static and dynamic activity. Tape induced changes in static foot posture paralleled those during walking and jogging.
O veruse injuries can occur in walkers and runners Lower extremity injuries constitute the majority of running injuries, and studies have estimated that $27-$ $70 \%$ of runners sustain overuse injuries during any one year period. ${ }^{1}$ Research suggests that some combination of abnormal structure and mechanics in the foot may increase the risk of injury. ${ }^{2-8}$ An example of a proposed predisposing factor to injury is the height of the medial longitudinal arch, which is often used as a surrogate, albeit indirect, measure of abnormal foot pronation. Williams et $a l^{3}$ reported that in low arched runners there is an increased prevalence of soft tissue injuries on the medial side of the lower extremity and at the knee, such as patella tendonitis, knee pain, and plantar fasciitis.

Several studies have investigated the effect of antipronation taping techniques on static foot posture and reported such techniques to be effective in controlling vertical navicular height (VNH). ${ }^{9-11} \mathrm{VNH}$, which is a measure of the medial longitudinal arch of the foot, decreases with pronation of the foot. In most previous studies of anti-pronation taping, VNH was measured in a stationary standing position as an index of whole foot pronation after the application of a taping technique and after a period of exercise. Although VNH has been shown to be reliable, ${ }^{9-13}$ it is our experience that the researcher requires a high degree of skill to be able to accurately identify the navicular tuberosity on repeated trials, such as before and after the application of tape. It is also a measurement that can only be easily taken in a stationary standing position, and in this study we were seeking to evaluate foot posture during walking and jogging. Furthermore, skin marker movement during gait is important and is responsible for a substantial error in measuring underlying position of bone, such as the navicular. ${ }^{14}$

Few studies have investigated the effect of anti-pronation taping techniques on dynamic measures of foot motion and posture-that is, the effect of anti-pronation taping during activity. A number of studies have used plantar pressure patterns as an indirect measure of foot pronation during walking, and found that LowDye taping medialised heel strike and anterior forefoot forces and diminished midfoot forces. ${ }^{15}{ }^{16}$ These researchers inferred from plantar pressures that LowDye taping provided support to the medial longitudinal arch, even though the relation between indices of plantar pressures and foot motion remains unknown. ${ }^{17}$

Although previous studies have provided valuable insight into the effect of taping techniques on static measures of foot alignment, they do not elucidate the effect of taping techniques on foot posture during activity. Several studies have attempted to explain the relation between static and dynamic lower extremity measures ${ }^{18} 19$ and have reported that such a relation is limited. There are two major deficits in our knowledge of the effects of anti-pronation taping on foot posture and motion. Firstly, the augmented LowDye taping technique, which has been shown to be superior to the LowDye taping technique in changing static measures of foot pronation, ${ }^{9}$ has not been evaluated dynamically - that is, foot posture during gait. Secondly, it remains unknown whether treatment induced changes in foot posture during standing reflect changes during gait. Thus the main aim of this study was to investigate the effect of an augmented LowDye taping technique on foot posture during dynamic tasks, such as walking and jogging. An additional aim was to evaluate the relation between tape induced changes in foot posture during standing and gait.

\section{MATERIALS AND METHODS}

This paper reports a repeated measures study which incorporated a cross over design in which participants served as their own control.

Abbreviations: $A H$, arch height; $A R$, arch height ratio; $M A$, metatarsal angle; $M C$, metatarsocalcaneal angle; $T L$, truncated foot length; $\mathrm{VNH}$, vertical navicular height 

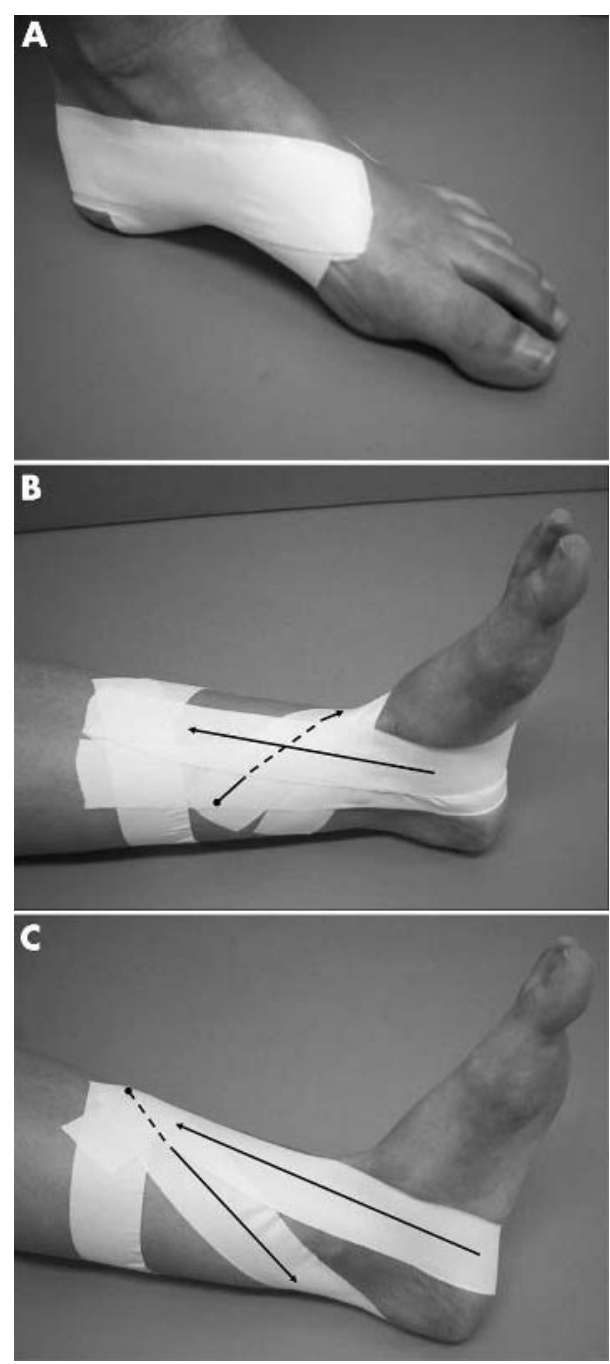

Figure 1 All taping techniques were applied with the foot in some supination and the first ray plantarflexed - that is, to ensure that the medial longitudinal arch is maximally arched. Participants actively held their foot in this position, while the therapist ensured that the position did not vary throughout the application. (A) LowDye technique. A spur is applied from the medial aspect of the neck of the first metatarsal and directed posteriorly around the back of the calcaneum to the lateral aspect of the neck of the fifth metatarsal. During application of this spur tape to the medial side of the foot, it is important to emphasise the plantarflexed position of the first ray and to ensure that the forefoot is slightly adducted. Mini-stirrups are then applied from the lateral aspect of the spur, running under the plantar surface making sure not to wrinkle the plantar skin, approximately perpendicular to the foot, and ending at the medial aspect of the spur. It is important to ensure that the supinated position of the foot is maintained throughout. (B) Reverse six technique. An anchor is applied one third up the length of the leg with application of a circumferential strip, making sure that the ankle is maximally dorsiflexed when this anchor is applied. The reverse six begins at the medial malleolus and courses laterally across the dorsum of the foot, under the midfoot in a lateral to medial direction, before crossing its origin to continue up to the anchor strip. Three were applied. (C) Calcaneal sling technique. Each begins from the anterior aspect of the anchor and courses distally in an oblique orientation towards the medial malleolus, passes under the midfoot and then over the posterior and lateral aspect of the calcaneum to course proximally and insert on its origin at the anchor strip. Two were applied. Consent was obtained for publication of this figure.

\section{Subjects}

Seventeen participants (five men, 12 women; mean (SD) age 27 (5.8) years) who performed regular fitness or athletic activity were recruited into the study. A difference in VNH greater than $10 \mathrm{~mm}$ when the foot was moved from relaxed calcaneal standing to subtalar neutral was required for inclusion in the study. Mueller et a ${ }^{20}$ suggest that a navicular drop in excess of $10 \mathrm{~mm}$ is abnormal and may contribute to foot pathology. VNH was measured using a Vernier caliper (Mitutoyo, Japan) in a procedure similar to that outlined by Vicenzino et al. ${ }^{9}$ Potential participants were excluded if they had coronary risk factors, a current lower limb injury requiring a decrease in activity or consultation with a health professional, had previously experienced anti-pronation taping, or had an allergic reaction to tape. After participants had been informed about the study, they were required to sign a consent form. The study was approved by the institutional review board.

\section{Apparatus}

A digital video camera (JVC GR-DV2000, UK), with a resolution of $720 \times 576$ pixels, was used to obtain images of the medial aspect of the foot and distal leg during standing, walking, and jogging tasks. The camera was fixed at the midpoint of an elevated $12 \mathrm{~m}$ runway and was not moved during data collection. The lens to foot distance was $2.6 \mathrm{~m}$, with the centre of the lens aligned approximately with the height of the medial malleolus. Images recorded on the digital video camera at 250 frames per second were then uploaded on to i-movie software (Apple Computers) on a G3 MacIntosh laptop computer (Apple Computers) with a capture rate of 30 frames per second.

\section{Treatment conditions}

The two treatment conditions for this study were the augmented LowDye tape ${ }^{9}$ and a no tape control. The augmented LowDye taping technique has been previously described and consists of the LowDye technique, involving spurs and mini-stirrups, with the addition of two calcaneal slings and three reverse sixes which are anchored on the distal third of the leg (fig 1). A rigid $38 \mathrm{~mm}$ wide sports tape (Leukosport; BDF, Sydney, Australia) with zinc oxide adhesive was applied to all participants by the same sports physiotherapist, who was experienced in the application of the technique.

\section{Experimental procedure}

Once qualified for the study, each participant was required to attend two sessions, the first of which was a screening and preparatory session and the second was the testing session.

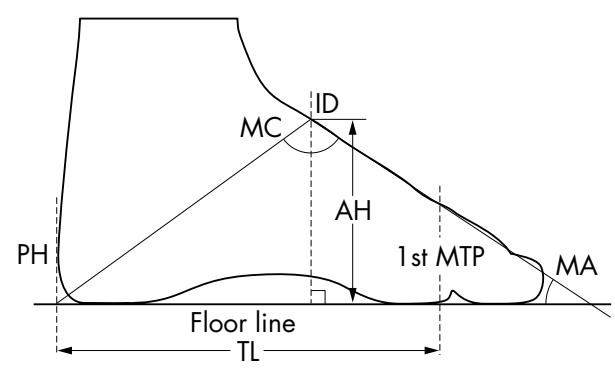

Figure 2 The indices of foot posture defined. Arch height ratio (AR) was calculated by dividing the arch height $(\mathrm{AH})$ by the truncated foot length (TL). The AH represented the height of the dorsal surface of the foot at a point (ID) projected perpendicularly from the floor line, midway along the length of the foot. The TL was measured from the posterior heel surface (PH) to the first metatarsophalangeal joint line (1 stMTP). The metatarsal angle (MA) was defined as the angle formed between a vector representing the floor line and a vector representing the dorsal surface of the forefoot. A metatarsocalcaneal angle (MC) was also calculated as the angle formed between the vector representing the dorsal surface and the vector representing the point of intersection of the $\mathrm{PH}$ with the floor line to the ID. 
In the first session the participant was examined to evaluate fit of inclusion/exclusion criteria. During the second session, a standard protocol was followed for testing. The foot with the greatest navicular drop was selected for tape application; the other foot acted as the control. The leg selected for taping was washed with soap and warm water to remove oil and dirt, and any hair in the region to be taped was removed to allow optimal adhesion of the tape. The first metatarsophalangeal joint line was identified by palpation and marked with an indelible pen.

Video footage was taken before application of the tape with the participant standing, walking, and jogging. While standing, the participant was positioned at the midpoint of the $12 \mathrm{~m}$ runway in stride stance. Video footage was collected across three trials. For the walking and jogging conditions, the participant was instructed to walk or jog over the $12 \mathrm{~m}$ runway at a self selected speed, which was monitored for consistency of foot placement on the platform across all recorded trials. The tape was then applied, and all measures were repeated. At the end of the testing session, the tape was removed with blunt nosed scissors, and the skin examined for adverse reactions.

\section{Determination of foot posture indices}

Video footage was edited to obtain static frames for the midstance phase of gait across condition, task, and time. The mid-stance phase was identified from the video footage as the middle frame between heel contact and toe off and was chosen because maximum pronation occurs at $55 \%$ of stance phase, ${ }^{21}$ and it was the phase of gait considered most comparable to standing. Five indices of foot posture were calculated from the digital images: truncated foot length $(\mathrm{TL})$, arch height $(\mathrm{AH})$, arch height ratio (AR), metatarsocalcaneal angle (MC), and metatarsal angle (MA) (fig 2). The measurements were calculated with a computer program developed in Microsoft Visual Basic and adapted from previous research in telerehabilitation. ${ }^{22}$ The software used the manual identification of landmarks, such as the first metatarsophalangeal joint, posterior calcaneum, anterior hallux, and the dorsum of the foot to perform linear distance and angular displacement measurements. The computer system was calibrated for this study and has been shown to produce reliable and valid biomechanical measurements in human subjects. ${ }^{23} 24$

\section{Reliability}

Acceptable intrarater and inter-rater reliability for the five indices of foot posture were determined by analysing digital images from the main study. A group of files was randomly selected to ensure inclusion of one of each of the independent variable combinations across the five dependent variables and was performed by two researchers. Table 1 presents the intraclass correlation coefficient and SEM for AR, TL, AH, MC, and MA. Except for MA, the reliability indices were above 0.8. Williams and McClay ${ }^{2}$ reported this calculation of AR to be the most reliable and valid measurement across two

Table 1 Inter-rater and intrarater intraclass correlation coefficients for foot measurements

\begin{tabular}{|c|c|c|}
\hline & \multicolumn{2}{|c|}{ Intraclass correlation coefficient } \\
\hline & Inter-rater & Intrarater \\
\hline Arch height ratio & $0.94(0.01)$ & $0.98(0.01)$ \\
\hline Arch height (mm) & $0.93(1.86)$ & $0.98(1.13)$ \\
\hline Truncated foot length (mm) & $0.96(1.2)$ & $0.98(0.93)$ \\
\hline Metarsocalcaneal angle $\left({ }^{\circ}\right)$ & $0.84(1.6)$ & $0.91(1.13)$ \\
\hline Metatarsal angle ( ${ }^{\circ}$ ) & $0.59(1.59)$ & $0.76(0.95)$ \\
\hline
\end{tabular}

Values in parentheses are SEM. weight bearing conditions ( $10 \%$ and $90 \%$ of weight bearing) compared with other measurements such as navicular height divided by foot length, navicular height divided by truncated foot length, and dorsum height divided by foot length.

\section{Data management and analysis}

Of the five dependent measures obtained (TL, AH, AR, MC, $\mathrm{MA}$ ), AR and MC were the only measures used for analysis. $\mathrm{TL}$ and $\mathrm{AH}$ were obtained for the calculation of $\mathrm{AR}$, and MA was excluded from further analysis because of poor reliability. Before analysis, measures were averaged across trials. Data are reported as means, $95 \%$ confidence intervals, and effect sizes (mean difference/pooled standard deviation). Three independent variables were incorporated into the research design: condition (tape, control), time (before and after application), and task (standing, walking, jogging). A three way, repeated measures analysis of variance (task by time by condition) was performed (using SPSS 11.0 for windows) to test the hypothesis that tape produced changes in AR in excess of control from before to after application for all tasks $(\alpha=0.05)$. Tests of simple effects were performed when significant interaction effects were present. If required, there would be six pair-wise comparisons to be evaluated with tests of simple effects. To correct for possible inflation of the type I error rate, a Bonferroni adjustment to the familywise $\alpha$ indicates the critical pair-wise $\alpha$ to be 0.0083 .

Pearson's correlation coefficients were calculated to evaluate the relation between the change in static and dynamic measures after application of the augmented LowDye tape.

\section{RESULTS}

\section{Effect of tape}

The results are presented in accordance with the two main questions of the study-that is, the effect of anti-pronation tape on dynamic measures of pronation and the relation between the effect of anti-pronation taping on static and dynamic measures of pronation.

Table 2 presents mean data and $95 \%$ confidence interval for $\mathrm{AR}$ and MC, both before and after taping across all tasks. The repeated measures analysis of variance identified a significant interaction effect for task by condition by time for AR $\left(F_{2,32}=6.394, \mathrm{p}=0.005\right)$ but not for $\mathrm{MC}\left(F_{2,30}=1.063, \mathrm{p}\right.$ $=0.358$ ). As can be seen in fig 3, the interaction effect is predominantly driven by the tape condition-that is, the difference after application from before-with task also playing a role-that is, AR is lower during jogging than during walking and standing.

Post hoc tests of simple effects revealed that there was no significant difference in mean AR between tape and control before application (standing, $\mathrm{p}=0.64$; walking, $\mathrm{p}=0.57$; jogging, $p=0.81$ ) across all tasks. After tape application, there was a significant difference in mean AR between tape and control conditions $(\mathrm{p}<0.002)$.

As illustrated by table 2 , the tape condition showed a mean increase in AR from before to after application of 0.031, 0.026, and 0.016 for standing, walking, and jogging respectively, whereas the control condition showed a mean decrease of approximately 0.001. Treatment effect sizes, between tape and control, before application were small $(<0.2)$ compared with after application treatment effect sizes $(\mathrm{AR}=1.72,1.11$, and 0.92 for standing, walking, and jogging respectively).

\section{Relation of static to dynamic measures}

The change in AR after taping measured during standing was correlated strongly with those measured while walking and jogging (Pearson's $r=0.7$ and 0.76 respectively; $\mathrm{p}<0.01$ ). 
Table 2 Arch height ratio (AR) and metatarsocalcaneal angle (MC) at mid-stance for condition (tape, control) and task (standing, walking, jogging)

\begin{tabular}{|c|c|c|c|c|c|}
\hline Index & Task & Time & Tape & Control & Condition difference \\
\hline \multirow[t]{9}{*}{ AR } & \multirow[t]{3}{*}{ Standing } & Before & $0.352(0.342$ to 0.361$)$ & 0.349 (0.339 to 0.360$)$ & $0.002(-0.007$ to 0.012$)$ \\
\hline & & After & $0.382(0.369$ to 0.395$)$ & $0.349(0.337$ to 0.361$)$ & $0.033(0.018$ to 0.048$)$ \\
\hline & & Difference & $0.031(0.023$ to 0.038$)$ & $0.000(-0.005$ to 0.003$)$ & \\
\hline & \multirow[t]{3}{*}{ Walking } & Before & $0.345(0.335$ to 0.336$)$ & $0.347(0.335$ to 0.360$)$ & $-0.002(-0.010$ to 0.006$)$ \\
\hline & & After & $0.371(0.359$ to 0.383$)$ & $0.347(0.335$ to 0.358$)$ & $0.024(0.013$ to 0.035$)$ \\
\hline & & Difference & $0.026(0.018$ to 0.033$)$ & $-0.001(-0.004$ to 0.002$)$ & \\
\hline & \multirow[t]{3}{*}{ Jogging } & Before & 0.329 (0.317 to 0.341$)$ & 0.330 ( 0.317 to 0.343$)$ & $-0.001(-0.011$ to 0.009$)$ \\
\hline & & After & $0.344(0.334$ to 0.355$)$ & $0.329(0.317$ to 0.341$)$ & $0.016(0.007$ to 0.025$)$ \\
\hline & & Difference & $0.016(0.007$ to 0.024$)$ & $-0.001(-0.004$ to 0.002$)$ & \\
\hline \multirow[t]{9}{*}{ MC } & \multirow[t]{3}{*}{ Standing } & Before & 125.05 (123.08 to 127.03$)$ & $125.40(123.60$ to 127.21$)$ & $-0.32(-1.82$ to 1.16$)$ \\
\hline & & After & $121.69(119.67$ to 123.71$)$ & 125.15 (122.98 to 127.31$)$ & $-3.74(-5.81$ to -1.67$)$ \\
\hline & & Difference & $-3.4907(-2.37$ to -4.61$)$ & $-0.0651(-0.97$ to -0.84$)$ & \\
\hline & \multirow[t]{3}{*}{ Walking } & Before & $125.14(123.04$ to 127.25$)$ & 124.99 (122.89 to 127.10$)$ & $-0.04(-1.04$ to 0.95$)$ \\
\hline & & After & 122.51 (120.54 to 124.48$)$ & 125.00 (122.95 to 127.04$)$ & $-2.49(-4.12$ to -0.85$)$ \\
\hline & & Difference & $-2.7411(-1.32$ to -4.16$)$ & $0.0034(-0.47$ to 0.47$)$ & \\
\hline & \multirow[t]{3}{*}{ Jogging } & Before & 127.09 (124.80 to 129.38$)$ & 126.50 (123.97 to 129.04$)$ & $0.46(-0.08$ to 1.73$)$ \\
\hline & & After & 125.02 (122.89 to 127.15$)$ & 126.71 (124.39 to 129.03$)$ & $-2.15(-3.92$ to -0.39$)$ \\
\hline & & Difference & $-2.3337(-3.79$ to -0.89$)$ & $0.2753(-0.23$ to 0.78$)$ & \\
\hline
\end{tabular}

Values are mean (confidence interval). A positive difference in mean AR corresponds to a decrease in foot pronation

\section{Adverse effects}

There were no adverse effects of the tape or experimental procedure on the participants.

\section{DISCUSSION}

The findings of this study support the hypothesis that antipronation taping is effective in reducing pronation as shown by changes in AR immediately after application. An increase in AR (AH divided by TL) could be produced by either an increase in $\mathrm{AH}$ and/or a decrease in TL, all of which feasibly correspond to a reduction in pronation. To our knowledge, this is the first study to show an anti-pronation effect of the augmented LowDye taping technique on dynamic measures during walking and jogging. Its findings support those found in previous studies ${ }^{9-11}$ that measured indices of pronation during standing — that is, statically.

The high intrarater and inter-rater reliability of the AR measurement strengthens the results. Furthermore, previous studies have supported the AR as a reliable and valid measure. ${ }^{2}$ The high reliability index along with the ability to selectively detect changes after the tape condition but not after the no-tape control condition suggests that AR may be an appropriate dynamic measure of pronation in the assessment of treatment effects.

The initial effects of anti-pronation taping on static and dynamic measures of pronation were highly correlated,

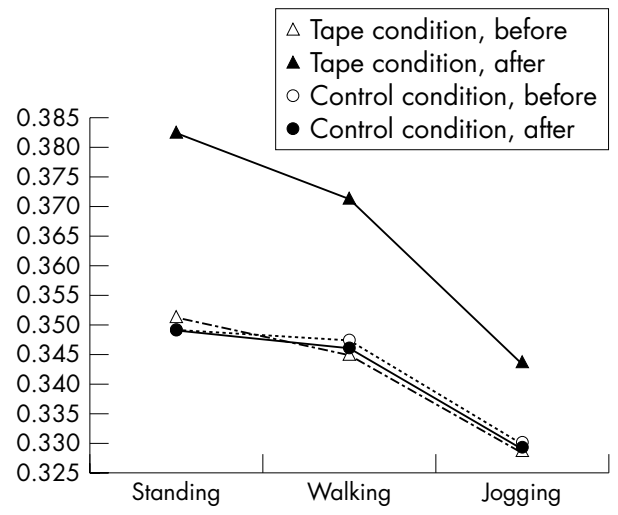

Figure 3 Condition (tape, control) by time (before, after) by task (standing, walking, jogging) interaction plot for arch height ratio at midstance $\left(F_{2,32}=6.39, \mathrm{p}=0.005\right)$.

\section{What is already known on this topic}

- The augmented LowDye anti-pronation taping technique, which is superior to the LowDye taping technique, has been shown to be effective in controlling static measures of foot posture (medial longitudinal arch height) after 20 minutes of jogging

\section{What this study adds}

- Video motion analysis showed that the augmented LowDye taping technique reduced pronation during walking and jogging

- This effect correlated with the effect of the tape on static alignment of the foot (standing)

suggesting a moderately strong relation between static and dynamic measures of AR. Future studies could therefore use static measurements of pronation, which would be simpler and less resource intensive during data collection and analysis.

It has been suggested that some combination of abnormal structure and mechanics in the foot, such as low arch height and pronation, may increase the risk of soft tissue injuries on the medial side of the lower extremity and at the knee. ${ }^{3}$ The findings from this study suggest that anti-pronation taping improves arch height and may therefore reduce the incidence of such injuries. Further work is warranted to evaluate such a possibility.

A possible limitation of the study is that it did not test the effect of taping on AR after 10 and 20 minutes of jogging as in previous studies. ${ }^{9-11}$ However, the moderate to strong relation between static (standing) and dynamic (walking, jogging) measures of change in pronation after taping suggests that the anti-pronation effect of the augmented LowDye tape after 10 and 20 minutes of jogging reported by Vicenzino et al, ${ }^{9}{ }^{10}$ who measured static arch height, would also be reflected by dynamic measures of pronation, although this notion requires validation in future research. 
In summary, the augmented LowDye tape significantly increased the arch height ratio during standing, walking, and jogging. This suggests that it is effective in controlling pronation during both static and dynamic activity. There was a moderately strong linear relation between static and dynamic measures of pronation, suggesting that research investigating the effect of anti-pronation taping on static measures may reflect tape induced effects during gait.

\section{Authors' affiliations}

B Vicenzino, M Franettovich, T Russell, G Skardoon, Department of Physiotherapy, University of Queensland, Brisbane, Queensland,

Australia

T McPoil, Northern Arizona University

Competing interests: none declared

Consent was obtained for publication of figure 1

\section{REFERENCES}

1 Hrejac A, Marshall RN, Hume PA. Evaluation of lower extremity overuse injury potential in runners. Med Sci Sports Exerc 2000:32:1635-41.

2 Williams DS, McClay IS. Measurements used to characterize the foot and the medial longitudinal arch: reliability and validity. Phys Ther 2000;80:864-71.

3 Williams DS, McClay IS, Hamill J. Arch structure and injury patterns in runners. Clin Biomech 2001:16:341-7.

4 Saltzman CL, Nawoczenski DA, Talbot KD. Measurement of the medial longitudinal arch. Phys Med Rehabil 1995;76:45-9.

5 Kaufman KR, Brodine SK, Shaffer RA, et al. The effect of foot structure and range of motion on musculoskeletal overuse injuries. Am J Sports Med 1999:27:585-93.

6 Tomaro JE, Burdett RG, Chadran AM. Subtalar joint motion and the relationship to lower extremity overuse injuries. J Am Podiatr Med Assoc 1996;86:427-32.

7 Holmes CF, Wilcox D, Fletcher JP. Effect of a modified, LowDye medial longitudinal arch taping procedure on the subtalar joint neutral position before and after light exercise. J Orthop Sports Phys Ther 2002;32:194-201.

8 Hintermann B, Nigg BM. Pronation in runners: implications for injuries. Sports Med 1998:26:169-76.

9 Vicenzino B, Feilding J, Howard R, et al. An investigation of the anti-pronation effect of two taping methods after application and exercise. Gait Posture 1997;5:1-5.

10 Vicenzino B, Griffiths SR, Griffiths LA, et al. Effect of anti-pronation tape and temporary orthotic on vertical navicular height before and after exercise. $J$ Orthop Sports Phys Ther 2000;30:333-9.

11 Ator R, Gunn K, McPoil TG, et al. The effect of adhesive strapping on medial longitudinal arch support before and after exercise. J Orthop Sports Phys Ther 1991;14:18-23.

12 Menz HB. Alternative techniques for the clinical assessment of foot pronation. J Am Podiatr Med Assoc 1998;88:119-28.

13 Evans AM, Copper AW, Scharfbillig RW, et al. Reliability of the foot posture index and traditional measures of foot position. I Am Podiatr Med Assoc 2003;93:203-13.

14 Tranber R, Karslson D. The relative skin movement of the foot: a 2-D roentgen photogrammetry study. Clin Biomech 1998;13:71-6.

15 Scranton PE, Pedegana LR, Whitesel JP. Gait analysis: alterations in support phase forces using supportive devices. Am J Sports Med 1982;10:6-11.

16 Russo SJ, Chipchase LS. The effect of LowDye taping on peak plantar pressures of normal feet during gait. Aust $J$ Physiother 2001;47:239-44.
17 Cornwall MW, McPoil TG. Reliability and validity of center-of-pressure quantification. J Am Podiatr Med Assoc 2003;93:142-9.

18 Cavanagh PR, Morag E, Boulton AJM, et al. The relationship of static foot structure to dynamic foot function. J Biomech 1997;30:243-50.

19 Hamill J, Bates BT, Knutzen KM, et al. Relationship between selected static and dynamic lower extremity measures. Clin Biomech 1989;4:217-25.

20 Mueller MJ, Host JV, Norton BJ. Navicular drop as a composite measure of excessive pronation. J Am Podiatr Med Assoc 1993;83:198-202.

21 Cornwall MW, McPoil TG. Motion of the calcaneus, navicular, and first metatarsal during the stance phase of walking. J Am Podiatr Med Assoc 2002;92:67-76.

22 Russell T, Jull G, Wootton R. Can the internet be used as a medium to evaluate knee angle? Man Ther 2003;8:242-6.

23 Russell T, Wootton R, Jull G. Physical outcome measurements via the internet: reliability at two internet speeds. J Telemed Telecare 2002;8(suppl 3):50-2.

24 Russell T, Wootton R, Jull G. Low bandwidth physical rehabilitation via the internet. J Telemed Telecare 2003;9(suppl 2):88-9.

\section{COMMENTARY}

The authors have presented a well designed study on the effect of the augmented low dye taping method on static and dynamic arch measurements. There are some aspects of the study that do not bear scrutiny, not the least of which is the repeated assertion that subtalar joint pronation (in particular), through its assumed but not proven coupling effect with other segments, is therefore a major mechanical contributor to lower limb overuse injury. The further assumption is that, if such motion can be "controlled", the injury may be treatable secondary to the mechanical change the tape imposes. This study establishes that the augmented low dye taping technique alters selected arch measurement parameters. It does not, however, in any way establish that the taping "controls" subtalar joint motion, nor that the mechanical change observed and measured has the ability to influence injury. Most studies in this area currently indicate that intervention at the level of the foot, be it through taping or an orthotic device, is achieved via a sensorimotor or psychophysical feedback loop, rather than by "motion control". ${ }^{12}$

S J Bartold University of South Australia, Health Sciences, South Australia, Australia; sbartold@internode.on.net

\section{REFERENCES}

1 Stacoff A, Reinschmidt C, Nigg BM, et al. Effects of foot orthoses on skeletal motion during running. Clin Biomech (Bristol, Avon) 2000;15:54-64.

2 Toyoda Y, Nigg BM, Wakeling JM, et al. Wavelet analysis can be used for evaluating correlation between vastus lateralis and vastus medialis for patients with patellofemoral pain syndrome. Proceedings of the 8th Annual Congress of the European College of Sports Science, 2003. 